\title{
EDGE DETECTION IN SEGMENTED IMAGES THROUGH MEAN SHIFT ITERATIVE GRADIENT $\mathbb{Z}_{n}$ USING RING
}

\author{
Esley Torres ${ }^{1}$, Roberto Rodriguez ${ }^{1}$, Yasel Garcés ${ }^{1}$, Osvaldo Pereira ${ }^{1}$ \\ ${ }^{1}$ Digital Signal Processing Group
}

Institute of Cybernetics, Mathematics and Physics (ICIMAF), Havana, Cuba

\begin{abstract}
In this paper, we propose a new method for edge detection in obtained images from the Mean Shift iterative algorithm. The comparable, proportional and symmetrical images are defined and the importance of Ring Theory is explained. A relation of equivalence among proportional images are defined for image groups in equivalent classes. The length of the mean shift vector is used in order to quantify the homogeneity of the neighborhoods. This gives a measure of how much uniform are the regions that compose the image. Edge detection is carried out by using the mean shift gradient based on symmetrical images. The difference among the values of gray levels are accentuated or these are decreased to enhance the interest region contours. The chosen images for the experiments were standard images and real images (cerebral hemorrhage images). The obtained results were compared with the Canny detector, and our results showed a good performance as for the edge continuity.
\end{abstract}

\section{KEYWORDS}

Edge detection, gray levels, ring theory, mean shift, gradient.

\section{INTRODUCTION}

Segmentation is a crucial step in any computer vision system, so much for the difficulties that this can have, as well as for the importance of its results. Basically, the segmentation can be regarded as a scene partition $\mathrm{g}$ in a set of non-overlapping regions $\mathrm{Ri}$, homogeneous with respect to some criterion whose union covers the entire image. In other words, the aim of the segmentation in the image analysis process is to separate the objects of interest of the rest, which is considered as background. Sometimes segmentation is often regarded as a process of classification of objects in a scene, and in certain measure a way of recognition of the same ones, since as the result of segmentation the different objects are located within the image (these seen as physical realizations of classes or abstract patterns). To carry out this classification is not trivial, because depend on the problem to solve and from the starting image. However, many segmentation techniques have been created, but the existence of a unique strategy that can solve all the encountered problems in the universe not exist yet. According to criterion of many authors the segmentation finishes when it satisfies the interests and goals of the observer. This is the reason why it is said that segmentation continues being an open problem yet. Many segmentation methods have been proposed for image data. Unfortunately, traditional segmentation techniques using low-level known as rigid methods, such as thresholding, histograms or other conventional operations, require a considerable number of iterations to obtain satisfactory results. 
Automation of these classical approximations is difficult due to the complexity in shape and variability within each individual object. Statistical criterion based on image segmentation has been an area of intense research activity during the past forty years and many algorithms were published in consequence of all this effort, starting from simple thresholding methods up to the most sophisticated random field type methods [9], [15], [1], [29], [31]. Unsupervised methods which do not assume any prior scene knowledge which can be learned to help segmentation processes are obviously more challenging than the supervised ones [27], [10].

The mean shift $(M S h)$ is an unsupervised non-parametric procedure that has demonstrated to be an extremely versatile tool for feature analysis. It can provide reliable solutions for many computer vision tasks. The mean shift method was proposed in 1975 by Fukunaga and Hostetler [6]. It was largely forgotten until Cheng's paper rekindled interest in it [2]. Unsupervised segmentation by means of the mean shift method carries out as a first step a smoothing filter before segmentation is performed [3], [4].

The Mean shift iterative algorithm (MShi), used in this paper, was proposed in 2006 and this has been utilized in many works by using the entropy as a stopping criterion.. The same has been introduced and applied in several previous works [19], [20], [21], [22], [23].

Edge detection is a crucial step of significant importance in image analysis. The purpose of edge detection is to identify regions of an image where a large change in intensity occurs. In literature have been reported many works related with edge detection which use spatial analysis of image. Despite the diversity of these methods there are two large groups in which may be separated: methods that employ global analysis [16] and those which use local analysis [28]. Local analysis being the more recommended for edge detection.

One of the limitations of the classical methods for edge detection is that they operate only in the range domain, i.e., with the pixel intensities. These methods are considered as rigid algorithms and in many of them the obtained results are edges with gaps. At the moment, most of the edge detector algorithms process the pixel information in the range-spatial domain, which is a good application for extracting features in the image.

The Ring Theory has been very used in cryptography and in many other applications in computer vision tasks [18], [30], [13]. However, few works have been concerned with edge detection by using the Ring Theory [17].

In 2013, the group theory was introduced for the spatial analysis in images [7] and later in [8]. The group theory was carried out considering the image like a matrix in which the elements belong to a cyclic group $\mathbb{Z}_{n}$. In this way, the image presents cyclical properties associated to the pixel values. This structure was used as a new stopping criterion for the Mean Shift Iterative Algorithm, where the same achieved a good performance. This means that the algebraic structures with cyclical properties are suitable for comparison among images. On the other hand, since rings are an extension of groups, the inclusion of the Ring Theory to the spatial analysis introduced in this paper is carried out in the same way used in [7]. The cyclical properties of the group are inherited for the ring, which will allow to increase or to diminish the difference among pixels values in a given region, and this difference will give us the way to find the edges in the analyzed image. In this work, we propose an approach for edge detection which involves the ring theory and we operate in the range-spatial domain. 
This paper continues as follows. In section 2, the most important theoretical aspects are exposed, some theorems are treated and their proofs are shown in appendix section. Specifically, in section 2.5, the Mean Shift Gradient Operator by using ring units for edge detection is introduced. Section 3 presents our algorithm for edge detection. In section 4, the experimental results and a comparison with the Canny Edge Detector are shown. In section 5, the most important conclusions are given.

\section{THEORETICAL ASPECTS}

In this section, the most important theoretical aspects corresponding to this study will be exposed, with the aim of understanding the analysis that will be carried out in the transformations of the images.

\subsection{Mean Shift Theory}

We first review the basic concepts of the Mean Shift Theory [6]. One of the most popular nonparametric density estimators is kernel density estimation. Given $n$ data points $x_{i}, i=1,2,3, \cdots, n$, in a neighborhood of radius $h$, drawn from a population with density function $f(x), x \in \mathbb{R}^{d}$ the estimated general multivariate kernel density at $x$ is defined by:

$$
f(x)=\frac{1}{n h^{d}} \sum_{i=1}^{n} K\left(\frac{x-x_{i}}{h}\right),
$$

By the use of theory and profile notation given in [2], the Mean Shift vector is given by

$$
\operatorname{MSh}_{h, G}(x)=\frac{\sum_{i=1}^{n} x_{i} g\left(\left\|\frac{x-x_{i}}{h}\right\|\right)}{\sum_{i=1}^{n} g\left(\left\|\frac{x-x_{i}}{h}\right\|\right)}-x
$$

where $g$ is the profile of $G$ and $K$ is a shadow kernel of $G$. The mean shift vector thus points toward the direction of maximum increase in the density. In other words, mean is shifted towards the region in which the majority of the points reside. Since the mean shift is proportional to the local gradient estimate, it can define a path leading to a stationary point of the estimated density, where these stationary points are the modes.

In [3], it was proved that the obtained mean shift procedure by the following steps, guarantees the convergence:

1) computing the mean shift vector $\operatorname{MSh}(x)$,

2) translating the window associated to the kernel.

Therefore, if the individual mean shift procedure is guaranteed to converge, a recursively procedure of the mean shift also converges. For more details about this algorithm see [5], [19], [20], [21], [22]. 


\subsection{The Ring $\mathbb{Z}_{n}$}

$\mathbb{Z}_{\boldsymbol{n}}$ is a partition of the set of integers $\mathbb{Z}$ in which the elements are related by the congruence module $n$ [11]. Mathematically speaking, we have :

$$
a \sim b \Leftrightarrow a \equiv b(\bmod n) \Leftrightarrow(b-a) \in n \mathbb{Z},
$$

where $\quad n \mathbb{Z}=\{0, n, 2 n, 3 n, \ldots\} \quad$ and $\quad n \in \mathbb{Z} \quad$ is fixed, $\quad \mathbb{Z}_{n}=\{0+n Z, 1+n \mathbb{Z}, 2+$ $n \mathbb{Z}, \ldots,(n-1)+n \mathbb{Z}\}$.

An abbreviated way is: $\mathbb{Z}_{n}=\{0,1,2, \ldots,(n-1)\}$, i.e., $\forall x \in \mathbb{Z}, x=\alpha+n \mathbb{Z} \Rightarrow x \in C_{\alpha}$, where $C_{\alpha}$ is the associated equivalence class to $\alpha$. The notation for the identity and unity of $\mathbb{Z}_{n}$ will be as 0 and 1 respectively.

Theorem 1: Generators of $\mathbb{Z}_{n}$

Let $n \in \mathbb{N}, \alpha \in \mathbb{Z}_{n}$ be such as $(\alpha, n)=1(\alpha$ and $n$ are relatively prime), then $\alpha$ is a generator of $\mathbb{Z}_{n}$

Theorem 2: Injectivity of generators of $\mathbb{Z}_{n}$

Let $\alpha, x, y \in \mathbb{Z}_{n}$ be $x \neq y,(\alpha, n)=1 \Rightarrow \alpha x \neq \alpha y$.

Theorems 1 and 2 show that multiplication by relative prime integers with $n$ is a way of bijective application over $\mathbb{Z}_{n}$. For this reason, one can obtain all the elements of $\mathbb{Z}_{n}$, only with the multiplication by an integer $\alpha$ such that $(\alpha, n)=1$.

If $(\alpha, n)=1$, then $\alpha$ and $n$ do not have any common divisor. The set $\left\{\alpha \in \mathbb{Z}_{n}:(\alpha, n)=1\right\}$ coincides with the set of units of $\mathbb{Z}_{n}$, by using the operation of multiplication congruence modulo $n$. These set will be denoted by $U\left(\mathbb{Z}_{n}, \cdot\right)$.

The system $\left(M_{m_{1} x m_{2}}\left(\mathbb{Z}_{n}\right), U\left(\mathbb{Z}_{n},\right) \cup\{0\}\right.$ establishes a vectorial space of all matrices of size $m_{1} x m_{2}$ over the field $U\left(\mathbb{Z}_{n}, \cdot\right) \cup\{0\}$. The particular interest is the subspace generated by a single image, and this for simplicity will be denoted by $F(G)$. An element of this subspace will denoted by $G_{j}$.

\subsection{Images via $\mathbb{Z}_{n}$}

Images are considered as a matrix in which each element is a vector component, which are non-negative real values, that belong to a discreet bounded interval which depends on the quantity of previously established bits. In particular, the images in gray levels will be the object of study, where its elements belong to $\mathbb{Z}_{+}$. However, the adaptation of the ring $\mathbb{Z}_{n}$ to images has the aim of incorporating the property of cyclic structure, characteristic that is not present on the images defined with $\mathbb{Z}_{+}$. Now, we will present the advantages using images with a ring structure and the significance of units of $Z_{n}$ for images.

Let $G$ be a gray level image, the presentation that uses 8 bit/pixel gives the possibility that each pixel can be represented until 256 gray levels in the interval [0; 255], i.e., $G \in$ 
$M_{m_{1} x m_{2}}(\mathbb{Z} \cap[0 ; 255])$. By multiplying the image by large numbers, the gray levels will stay with the maximum value of the aforesaid range. Of a similar way, this happens with addition. By the features of $\mathbb{Z}_{n}$, one can take advantage of its cyclic properties. However, in the current shape in that the images are defined, this is not favorable for our purpose, (see Figure 1). For this reason, the image is redefined in the following way. Let $\mathrm{G}$ be an image like a matrix in which the elements belong to the ring $\mathbb{Z}_{n}$, i.e., $G \in M_{m_{1} x m_{2}}\left(\mathbb{Z}_{n}\right)$. As was pointed out previously, the main property acquired by the images, defined in this way, it is the cyclical property under addition and product operations. This property is shown in Figure 2, where it is represented what happens to the gray level values when the addition and multiplication are carried out.

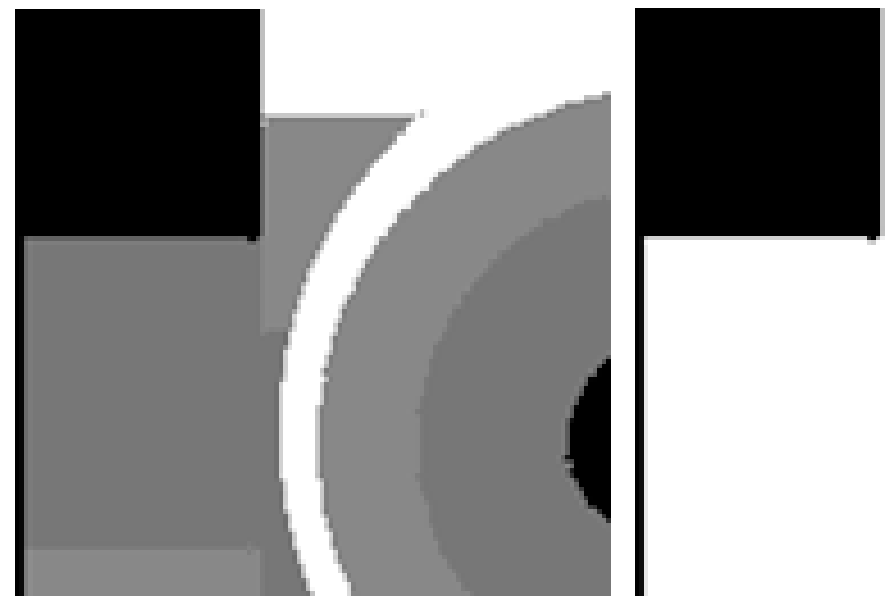

(a)

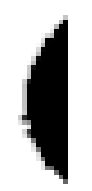

(b)

Figure 1. Effect of multiplying gray level image by a number. (a) Original image, (b) original image multiplied by a large value.

Figure 2 represents an image that characterizes the addition and the multiplication of a gray levels vector in the interval $\left[0,2^{B}-1\right]$, in this case $B=8$. One can note that when the operation (addition or multiplication) is carried out with big values, the result in the image greater than 255 , it is always forced to be 255 . This fact is independently of the values that were used to carry out the operation, and it is a consequence of the necessity of preserving the values of the pixels in the preset interval. Figure 2(a) shows the addition of that vector with the numbers $+0 ;+1 ;+2 ; \cdots ;+255$. In Figure $2(b)$ is represented the product of that vector with the previously mentioned numbers. Note that, one of the main advantages that has the group $\mathbb{Z}_{n}$, it is that when one operates with any two elements of $\mathbb{Z}$ the result is in the interval $\left[0,2^{B}-1\right]$, i.e., only takes positive values. This characteristic is favorable if we considered the intensities of gray levels like elements of the ring $\mathbb{Z}_{n}$, because negative values are not generated when operates in this set, and therefore, the previous situation is avoided. In Figures 2 (c) and 2(d) are shown the same mentioned operations in ring $Z_{n}$. Note that those values exceeding 255 are forced to the interval $[0,255]$, by congruence module $n$ operation. In Figures 2(c) and 2(d), one can see that, in the multiplication, the changes of the gray values are more emphasized than in the addition. 

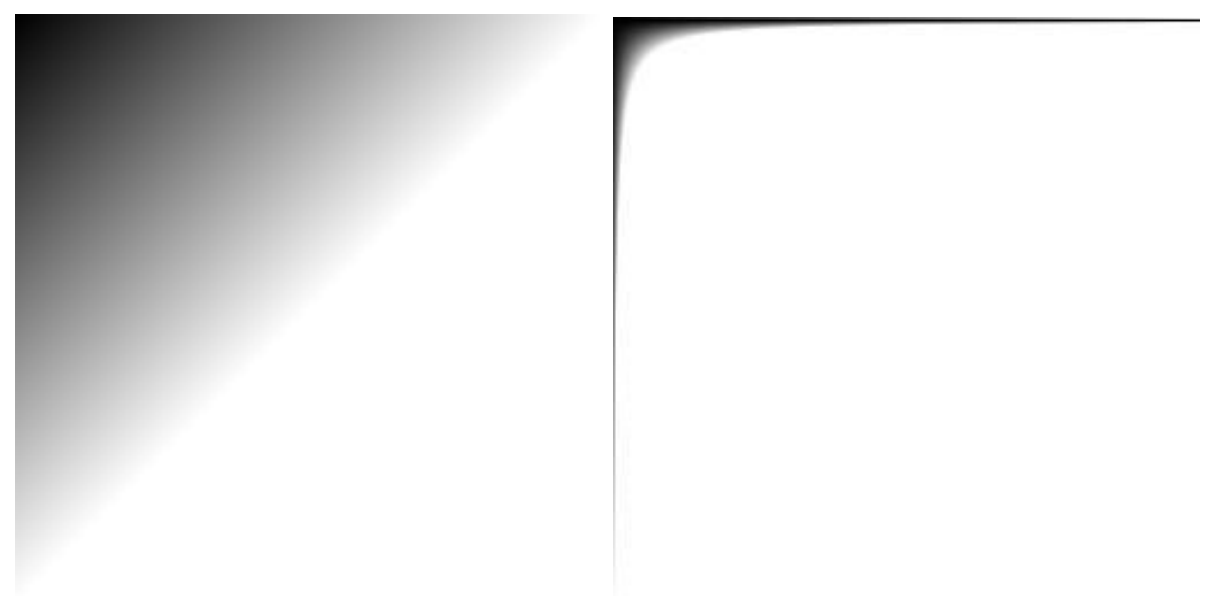

(a)

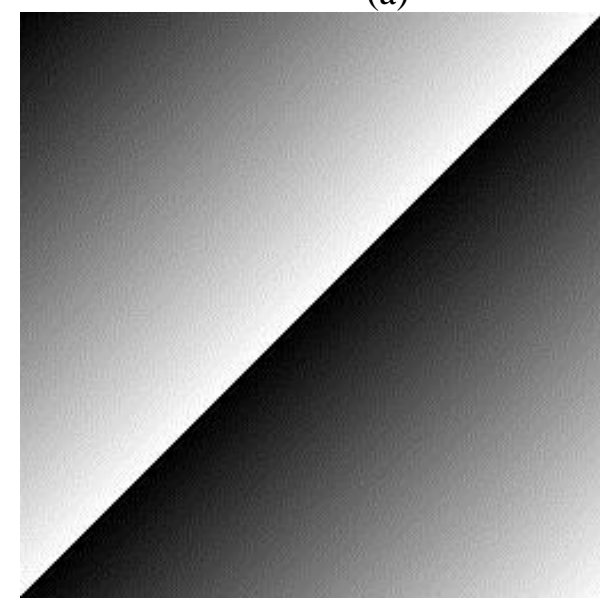

(c)

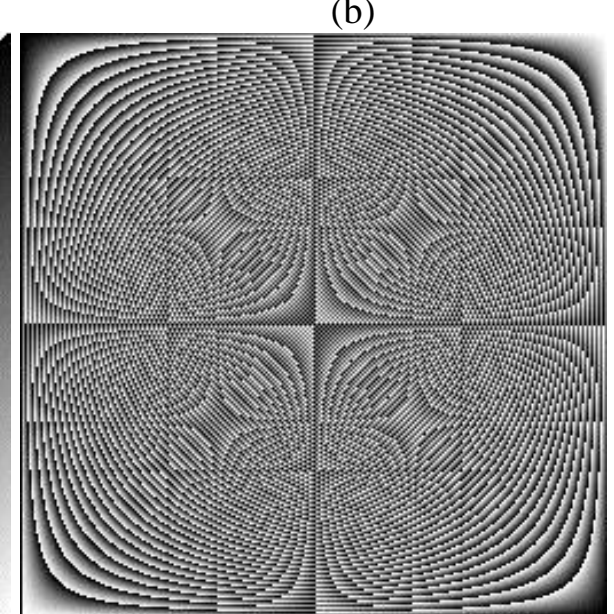

(d)

Figure 2. Effect on the gray levels when add and multiply by different values. $X$-axis: gray levels vector in the interval $\left[0,2^{B}-1\right], Y$-axis: gray levels vector in the interval $\left[0,2^{B}-1\right]$ by using the numbers from $+0,+1,+2, \cdots,+255$. (a) Addition and coefficients in $\mathbb{Z} \cap[0,255]$, (b) multiplication and coefficients in $\mathbb{Z} \cap[0,255]$, (c) addition and coefficients in $\mathbb{Z}_{n}$, (d) multiplication and coefficients in $\mathbb{Z}_{n}$.

\subsection{Comparable and Proportional Images}

Definition 1: Comparable images

In the set of images, we will say that two images are comparable if they have the same dimension, i.e.:

Let $G_{1}$ and $G_{2}$ be two images and size $m_{1} \times m_{2}$ and $n_{1} \times n_{2}$ respectively, then it is said that are comparable iff $m_{1}=n_{1}$ and $m_{2}=n_{2}$.

Definition 2: Proportional images

Let $G_{1}$ and $G_{2}$ be two comparable images, then it is said that $G_{1}$ and $G_{2}$ are proportional if exists $\alpha \in \mathbb{Z}_{+}$such that $G_{1}=\alpha * G_{2}$, where $(\alpha, n)=1$, i.e., $G_{1}(i, j)=\alpha * G_{2}(i, j)$.

Theorem 3: Equivalence relation of proportional images 
Let the symbol $\sim$ represent the relation defined on a set of comparable images such that $G_{1} \sim G_{2}$ are proportional. Then, the relation between $G_{1}$ and $G_{2}$ is an equivalence relation.

Two cases in that the images are proportional, $\alpha$ and $n$ being not relatively prime integers, takes place when the images are totally homogeneous, or when all the elements that compose the image (pixels) and $n$ are relatively prime integers. In the first case, we are not interested, and the second is not very usual in real images. If the image $G$ is such that all its elements are zeros, then only is proportional to itself. Therefore, if $(\alpha, n) \neq 1$, then is not always ensured the proportionality among images.

Theorem 4: Bijective correspondence of proportional images

If two images are proportional, then their respective sets of gray levels are on bijective correspondence.

The previous theorem specifies that if two images are proportional, then exists a bijective function between them given by the product of the image and a number $m$ such that $(m, n)=$ 1.

Theorem 5: Entropy of proportional images

If two images are proportional then have the same entropy.

Definition 3: Relative Symmetrical Image

Let $G, G_{1}$ two proportional images and $\alpha \in Z_{n}$ be, such that $G_{1}=\alpha * G$. It is said that an image $G_{2}$ is the relative symmetrical image of $G_{1}$ with regard to $\mathrm{G}$ by using parameter $\alpha$ if $G_{2}=\alpha^{-1} * G$, where $\alpha^{-1}$ is the additive inverse of $\alpha$.

To avoid confusions with the signs of the subtraction operations and subindex notations, starting from this moment to make reference to the symmetrical unit of $\alpha$, it will be used $\alpha^{-1}$ to an scalar element, and $-\alpha$ when it is about to a subindex. Note that $\alpha+\alpha^{-1}=0$. The pair of symmetrical images will be denoted by $G_{\alpha}$ and $G_{\alpha^{-1}}$.

Note that the values $\alpha$ and $\alpha^{-1}$ are symmetrical regarding the ring center value. The visual effect in $G_{\alpha}$ and $G_{\alpha^{-1}}$ is that an image is the negative regarding the other one. The result by product of units has several purposes in depending on the used unit and the image. For example, for $\alpha=1$ we obtain the classical negative image (see Figure 3(a) and 3(d)). For values between 3 and 7 we can highlight the contour for some objects that compose the image (see Figures 3(c) and 3(e)). These values will be used in the next sections for edge detection. However, for large values; for example, greater than 40 , the image becomes very noisy (see the third column in the Figure 3). Previous research gave that values greater than 7 make a distortion on contours. For this reason, units values to preserve contours, are on the interval $[1,7]$ These effects will be studied in next researches. 
International Journal of Soft Computing, Mathematics and Control (IJSCMC),Vol. 4, No. 2, May 2015

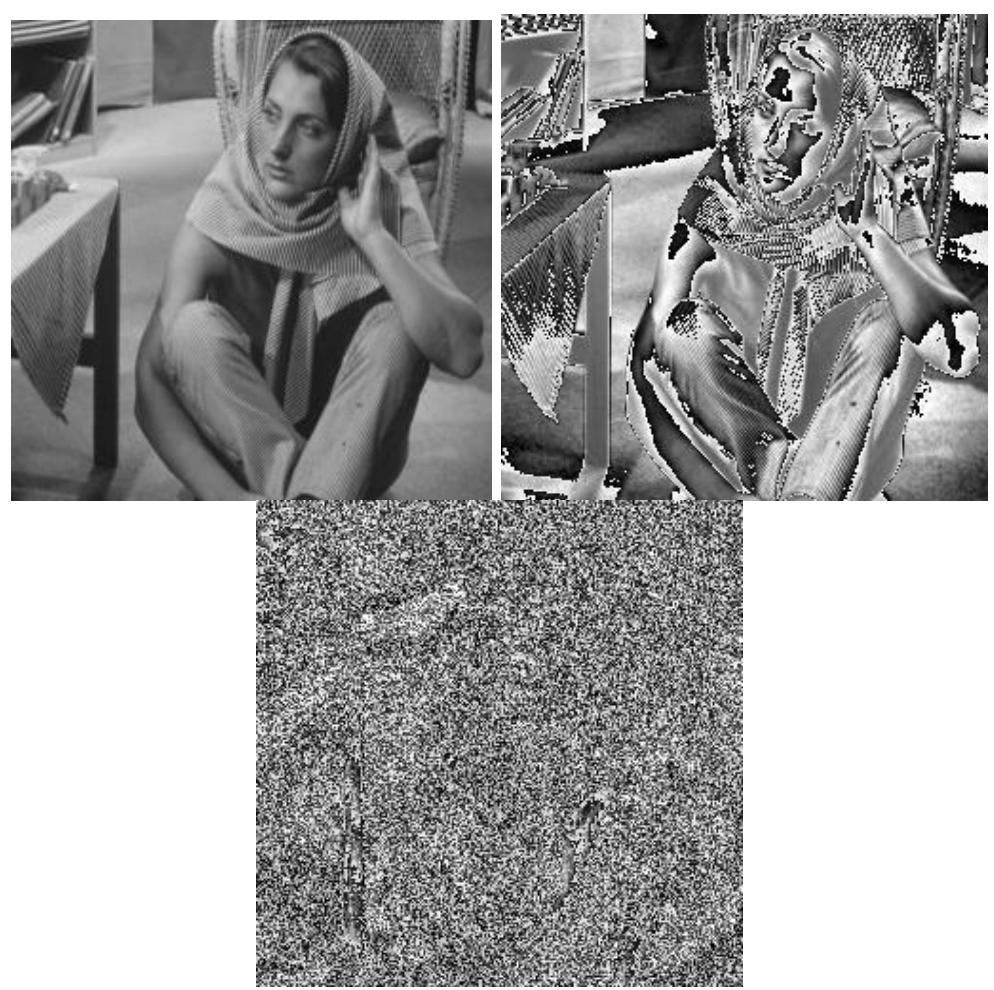
(a) $\alpha=1$
(b) $\alpha=3$
(c) $\alpha=65$

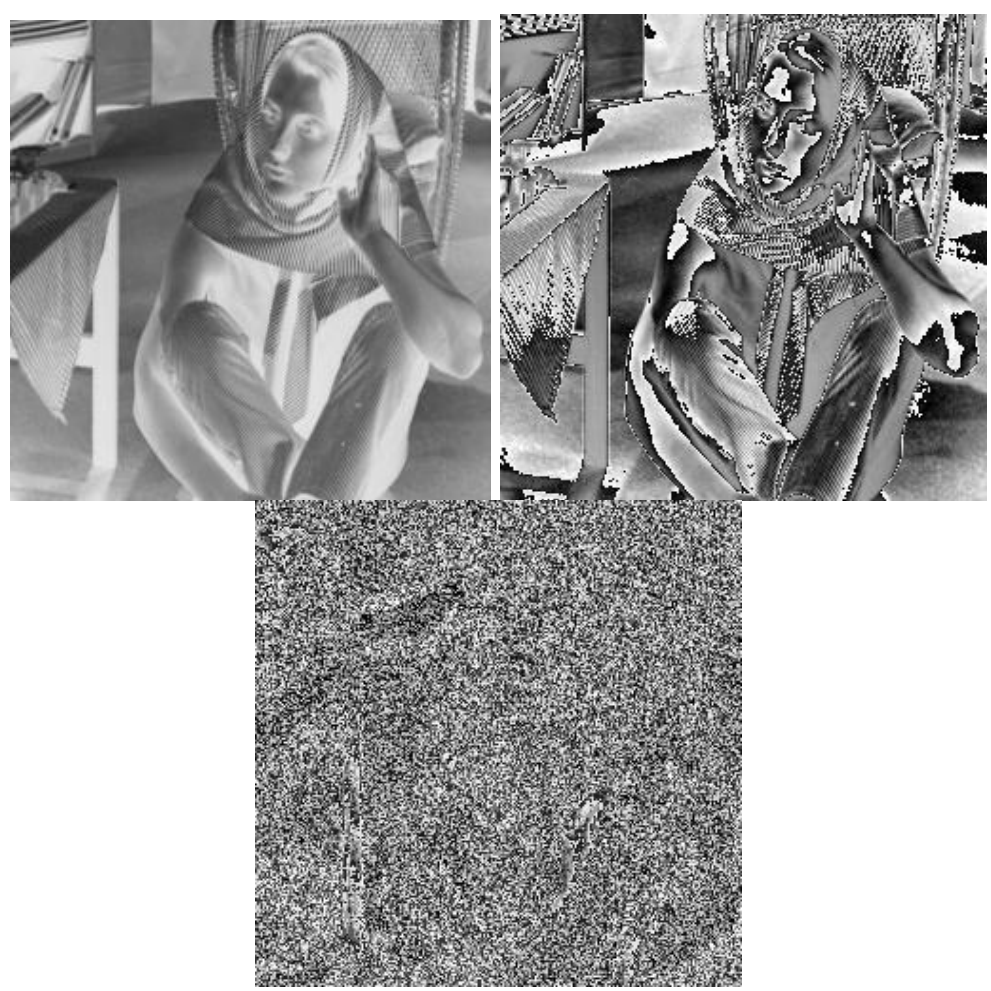
(d) $\alpha=255$
(e) $\alpha=253$
(f) $\alpha=191$ 
Figure. 3. Symmetrical images. First row: product by small units $\alpha$. Second row: product by $\alpha^{-1}$ (symmetrical unit of $\alpha$ ).

\subsection{The gradient operator of the Mean Shift using symmetrical units}

In the previous section, the main properties of the units of the rings were exposed with the multiplication operation. In this section, we combine these properties with the Mean Shift in order to produce an image gradient.

Definition 4: Mean Shift of an image

Let $G$ be an image, the image that computes the Mean Shift procedure at each point is called the mean shift of $G$. Notation for this application is $M S h(G)$.

By means of the use of ring units, we use a special notation when Mean Shift vector is applied to an image $G$ that has been multiply by ring units. This element is denoted by $M S h_{\alpha}(G)$, it is given by the following equation:

$$
\operatorname{MSh}_{\alpha}(G)=\operatorname{MSh}(\alpha * G)
$$

Definition 5: Mean Shift Gradient Operator by a Ring Unit

Let $\mathrm{G}, \alpha$ be an image and a ring unit respectively, the Mean Shift Gradient Operator of $\mathrm{G}$ by using $\alpha$, denoted by $M G R U_{\alpha}(G)$ (Mean Shift Gradient by Ring Units), is defined as:

$$
M G R U_{\alpha}(G)=\operatorname{MSh}_{\alpha}^{2}(G)+M S h_{-\alpha}^{2}(G)
$$

In Figure 4, it is shown the image gradient by using $M G R U_{\alpha}$ applied to a naive binary image made up by a square, where $\alpha=1$. Note in the first row of this figure, the $M S h_{\alpha}(x)$ and $M S h_{-\alpha}(x)$. direction points out from highlighted regions toward dark regions. This is the reason why $M S h_{\alpha}(x)$ describes the density from outside toward inside the square, while happens on the contrary with $M S h_{-\alpha}(x)$, which describes the density from inside toward out. To see better the behavior of the MSh gradient, in the second line of Figure 4 are shown, in a quantitative way, the zooms of the superior left corner of the square, which correspond respectively, to the images of the first line. 
International Journal of Soft Computing, Mathematics and Control (IJSCMC),Vol. 4, No. 2, May 2015
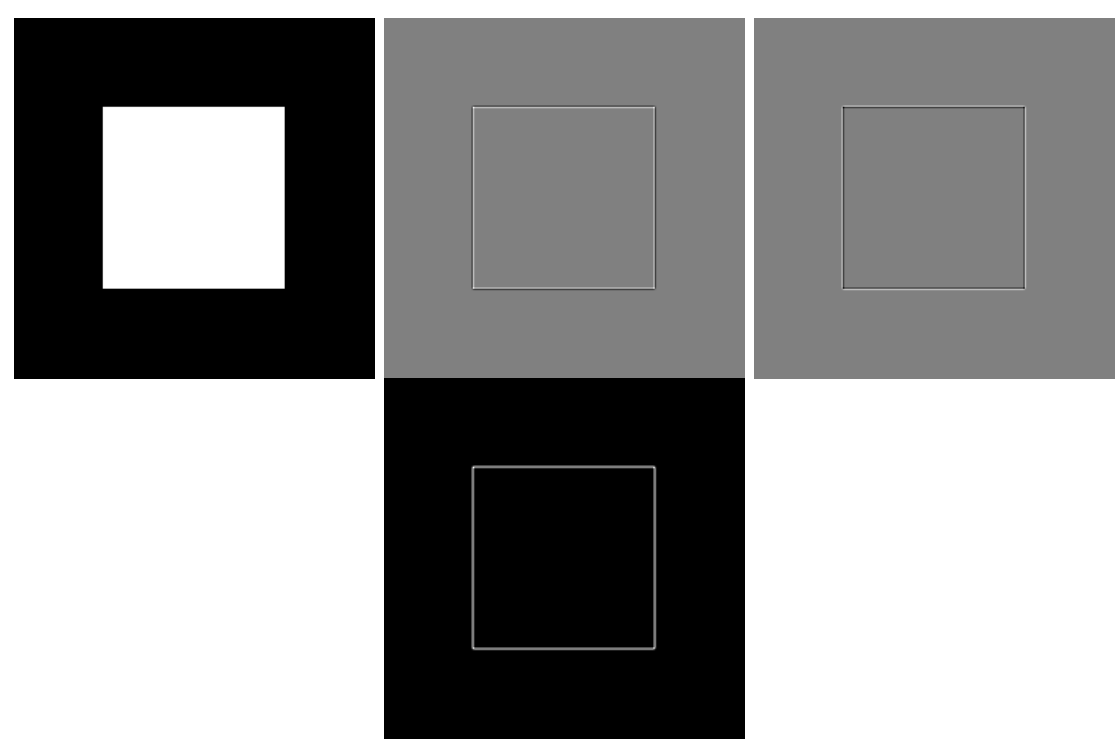

(a) Original image

(b) MSh1

(c) MSh255

(d) MGRU

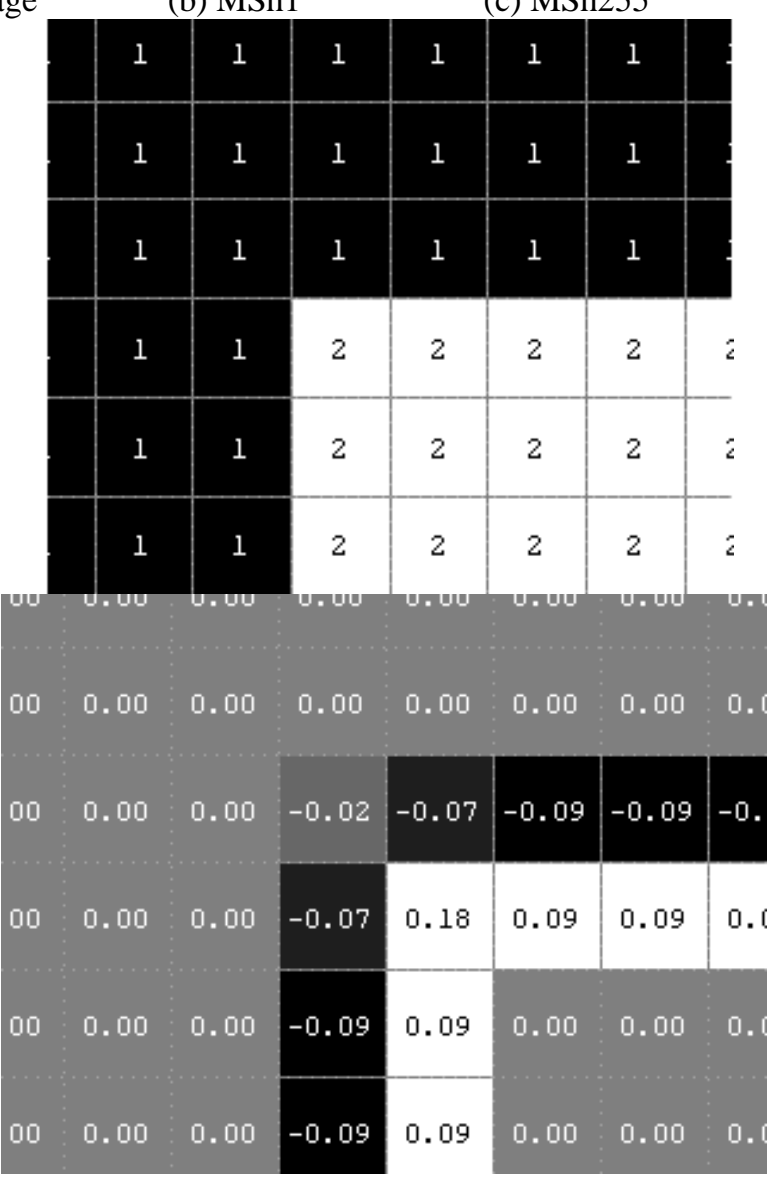

(e)

(f) 
International Journal of Soft Computing, Mathematics and Control (IJSCMC),Vol. 4, No. 2, May 2015

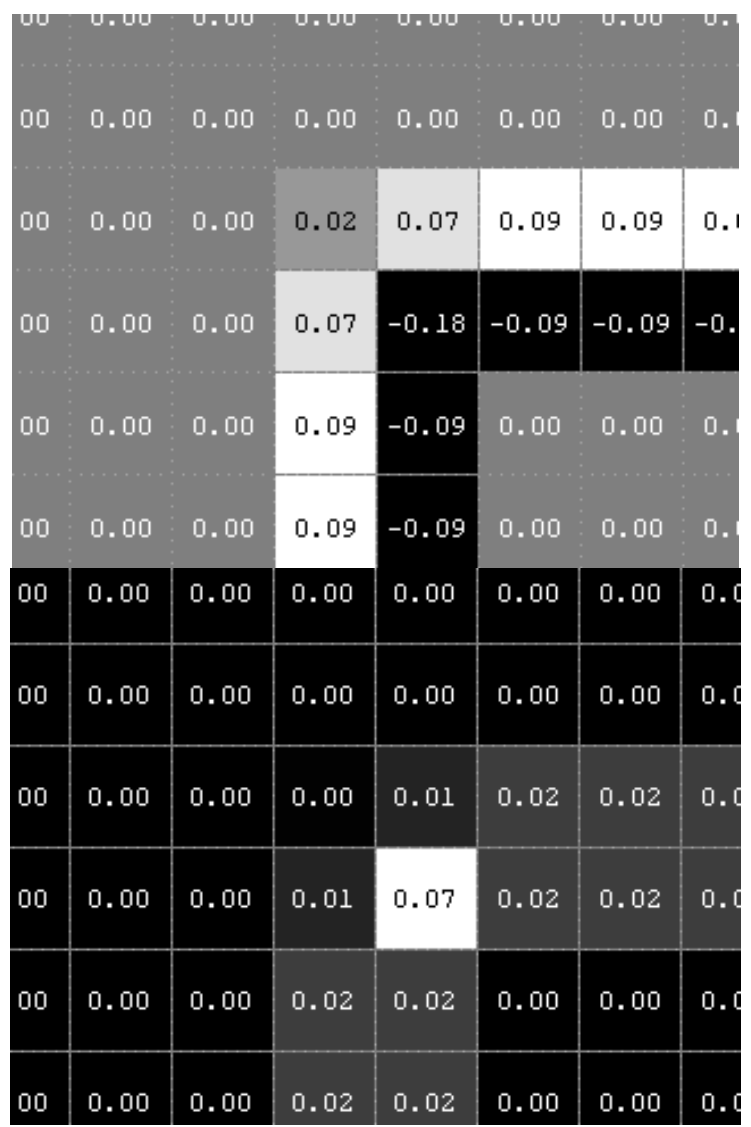

$(\mathrm{g})$

(h)

Figure 4. First row: Images in natural size. Second and third row: increased images (zoom).

Note that, in Figures 4(h), 4(f) and 4(g), MSh values are 0 for those pixels that belong to homogeneous neighborhood. This means that, in the neighborhood of these pixels every neighbor has the same gray level of the central pixel. On the other hand, the zoom image applying MGRU is shown in Figure 4(h). The difference among pixel values that belong to a border and those that not, it is so remarkable that the borders are highlighted. The resulting image highlights the edges of the square.

\section{ALGORITHM}

In this section, we present our algorithm of the Mean Shift Gradient Operator using Ring Unit $(M G R U)$. Our algorithm is constituted by three main steps:

1) Filtering

2) The use of Ring Units

3) Thresholding.

Filtering step is important when real images are processed, in this case, we remove noise from image. The use of ring unit is the most important step. In this step, the contour of objects are moved toward outside. Here, the unit parameter is denoted by $\alpha$. On the other hand, the thresholding step is necessary in order to enhance the edges. The parameter related to this step 
is denoted by t. The following algorithm shows the general structure of our strategy for edge detection:

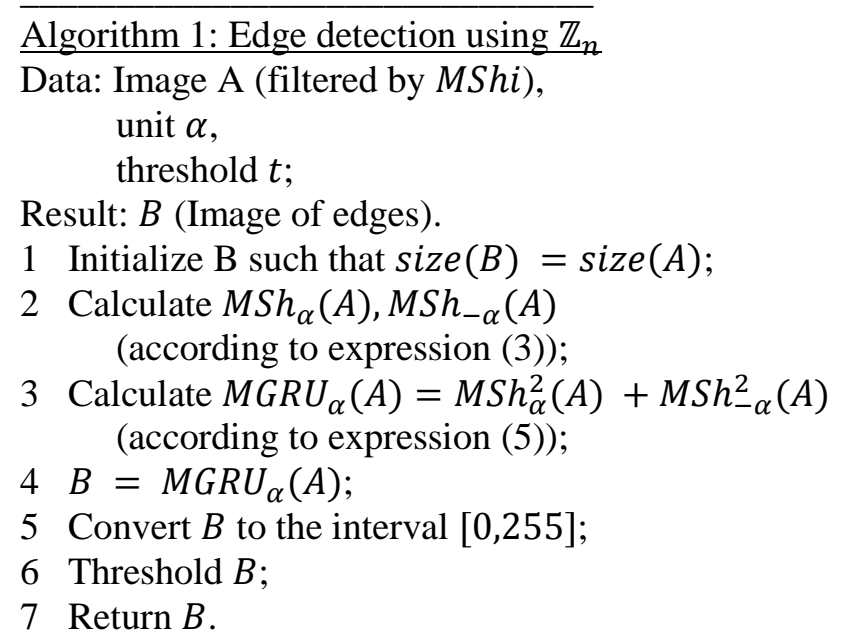

\section{EXPERIMENTAL RESULTS}

In this section, we show the experimental results by applying our algorithm. We consider for the experiments two kind of images, the obtained manual segmentation (groundtruth) of the Berkeley database and real images (cerebral hemorrhage images). Attending for each type of image features, we carried out the selection of parameters. For this reason, the parameters were chosen according to a previous analysis of the studied images. Automation of these parameters will be of study in future works.

For the groundtruth image is not necessary the filtering step. The smallest nonzero value that can be selected from the interval is assigned to the threshold parameter t. Thus, every pixel with value greater than zero is considered as an edge. As the regions are totally homogeneous, it is not needed to highlight a specific region. The results are compared with the Canny Edge Detector. On the other hand, real images are treated of a different way. The filtering process is carry out by a few iterations of the Mean Shift Iterative Algorithm, as it was described. in section 2.5 The parameters for this step are as follows: $\mathrm{hs}=10, \mathrm{hr}=10$, eds $=0: 9$ (stopping criterion for the Mean Shift Iterative Algorithm). In order to avoid undesirable results, white matter has been removed from the image, the method to remove white matter is shown Figure 5(a), using the image profile crossing the hemorrhage by a thresholding algorithm. The image profile, also give us the key to choose the appropriated ring unit. Note in figure 5(c), the gap between gray matter and hemorrhage, that it is indicated by a discontinuous line. The key idea for our purpose, it is to make that this contour reaches a large difference of gray levels. This is because, we need to emphasize a big difference between gray matter and hemorrhage. According to previous section the ring unit must belong to the interval $[1,7]$, to preserve contours. The selection $\alpha=3$, shows a good behavior for edges, that it is shown in Figure $5(\mathrm{~b})$. Figure 5(d) shows the image profile of 5(d), note that the gap between gray matter and hemorrhage has been reached a big difference compare with image 5(b). 
International Journal of Soft Computing, Mathematics and Control (IJSCMC),Vol. 4, No. 2, May 2015

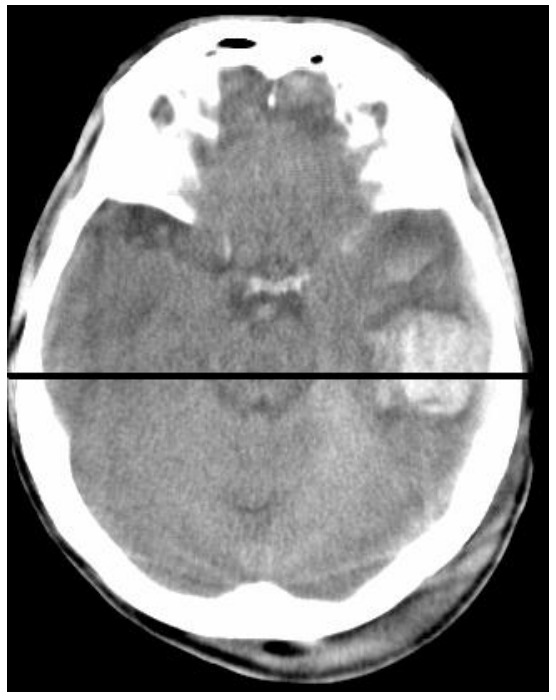

(a)

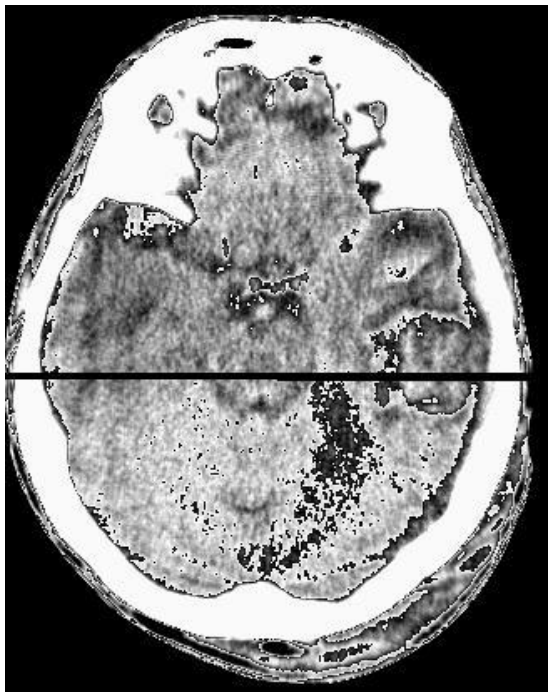

(b)

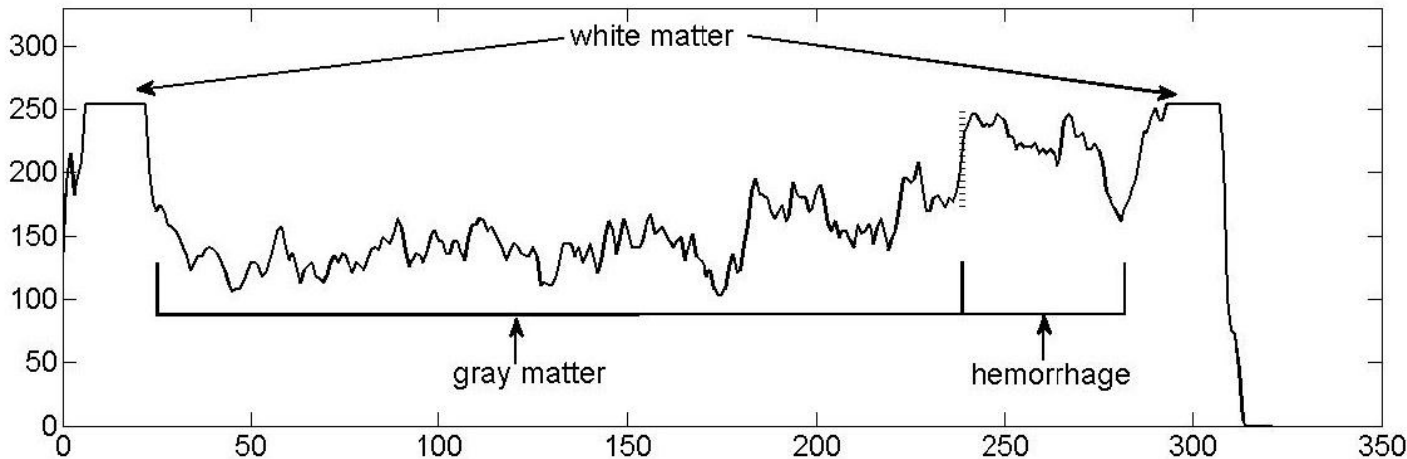

(c)

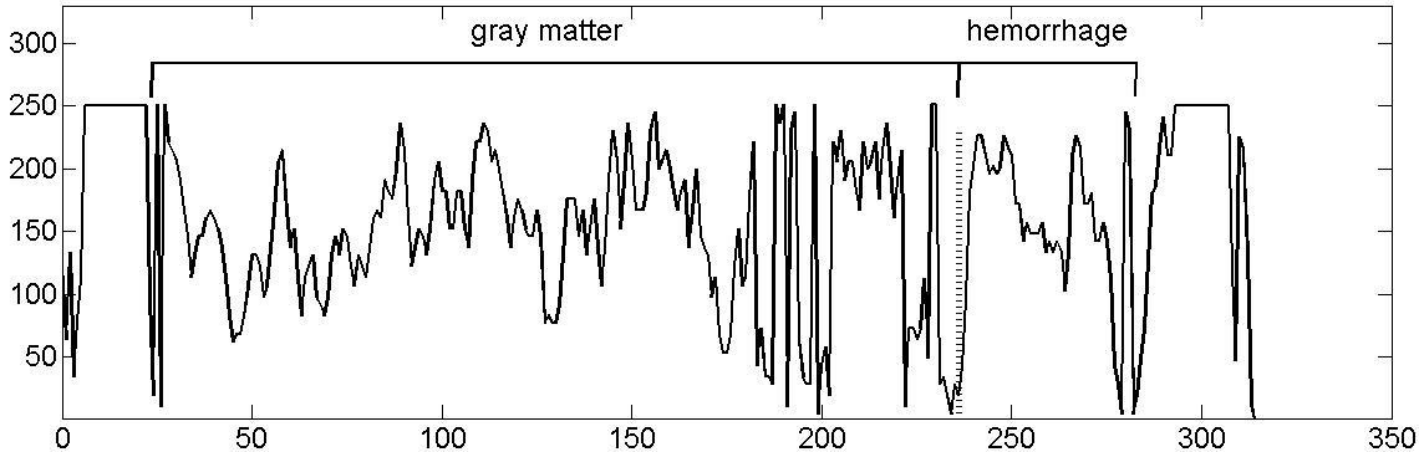

(d)

Figure 5. Image profile. (a) Cerebral hemorrhage image. (b) Intensity Profile.

Figure 6 shows the result of applying the Mean shift Gradient. Note that multiplying by the appropriate unit, in this case $\alpha=5$, the effect of preserve contours have been reached successfully. 
International Journal of Soft Computing, Mathematics and Control (IJSCMC), Vol. 4, No. 2, May 2015

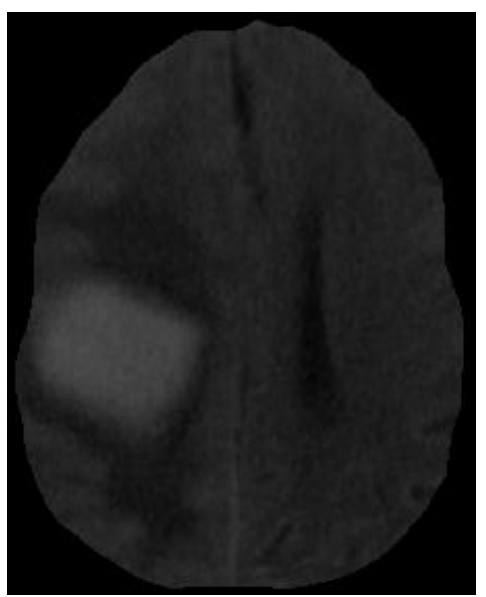

(a) Original image

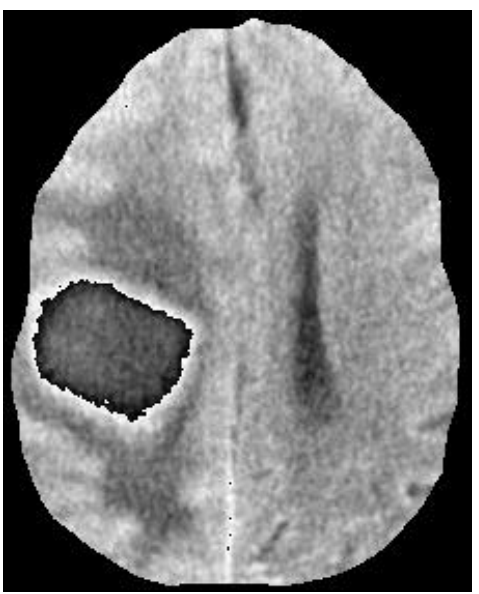

(b) $\alpha=5$

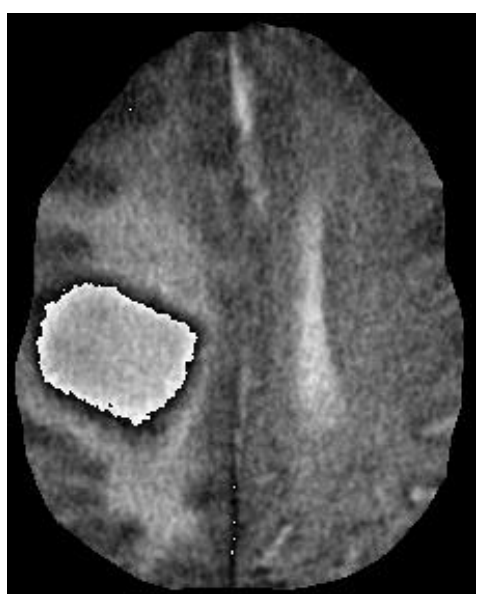

(c) $\alpha=251$ 
International Journal of Soft Computing, Mathematics and Control (IJSCMC),Vol. 4, No. 2, May 2015

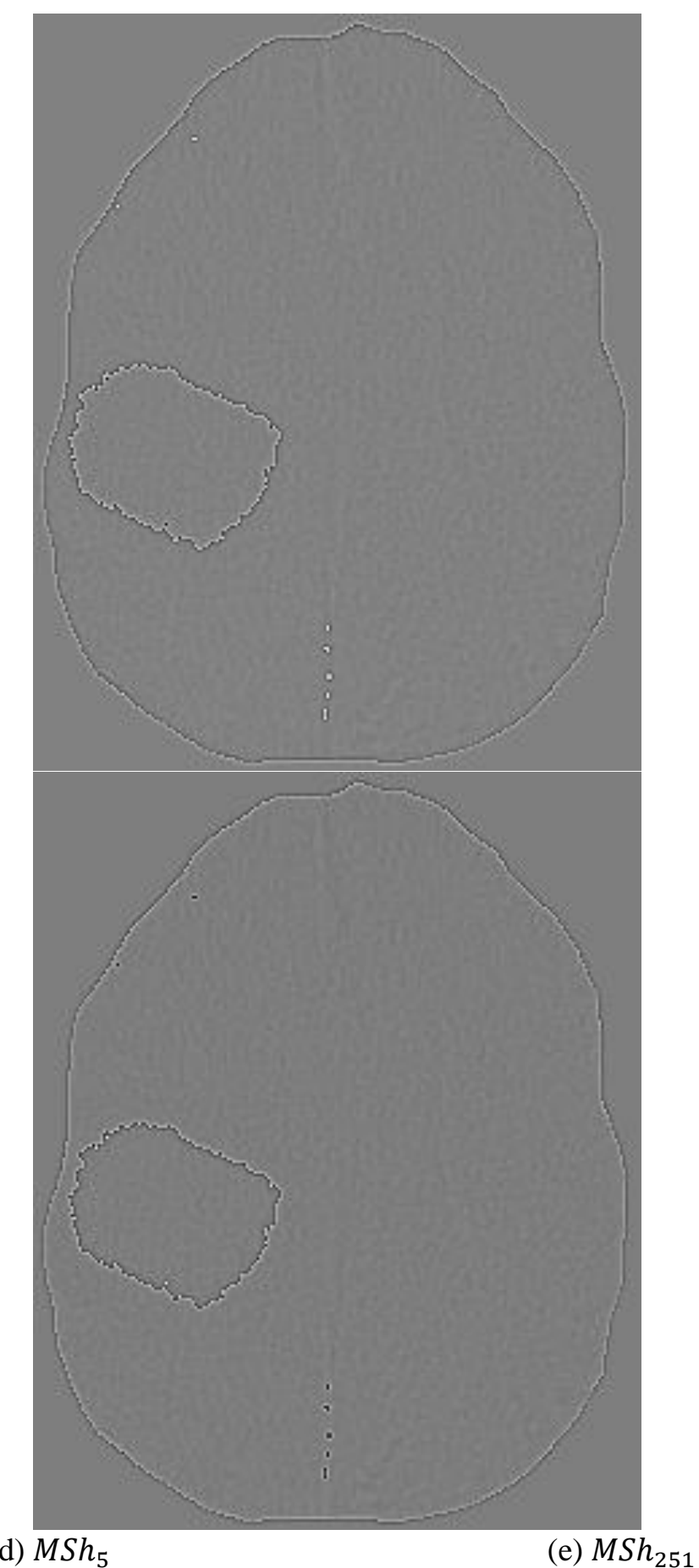

Figure 6. Effect of multiplying by symmetrical units (no filtering step).

The threshold step is given in order to remove the gray levels that are not associated to edges from the MSh gradient image. The interval size of the MSh gradient values is too large, oscillating between 104 and 106. Thus, we proportionally have reduced this to [0;255]. In Figure 7(a) is shown a histogram of a MSh gradient image of a cerebral hemorrhage image. The arrow placed on 30 value, divides the Figure in two regions. The region with values by below 30, represents the probabilities occurrence of pixel with gray level less than 30 , these pixels belong to background. The other region is related to edges. The threshold parameter $t$ 
International Journal of Soft Computing, Mathematics and Control (IJSCMC),Vol. 4, No. 2, May 2015

for this image is precisely 30 . All the other used brain images in this work have a similar behavior. The results before the thresholding process and after this have a significant difference (see Figure 7(c) and 7(b)).

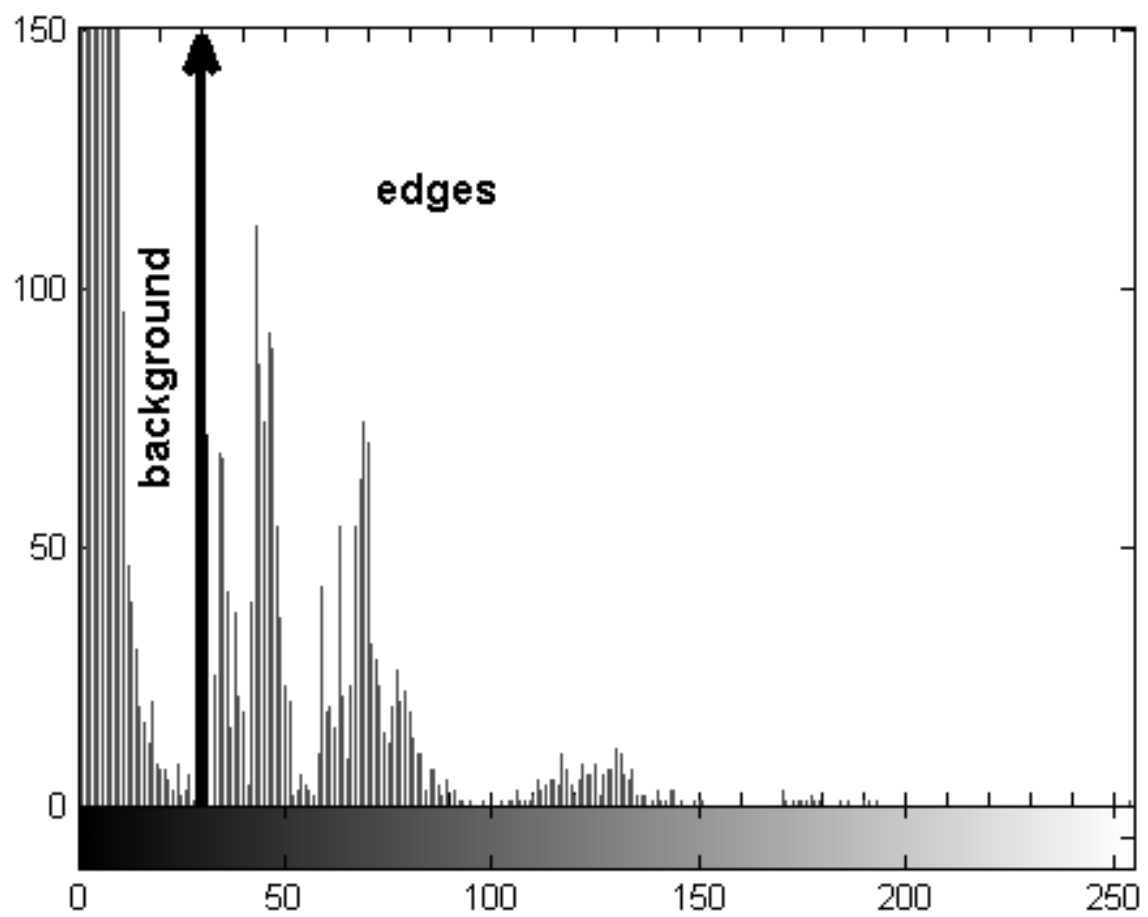

(a)

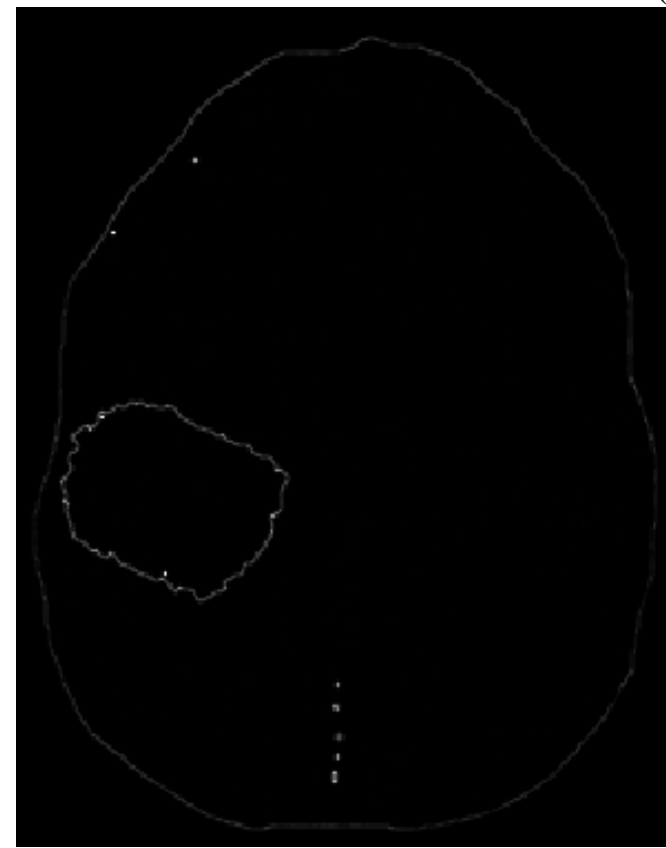

(b)

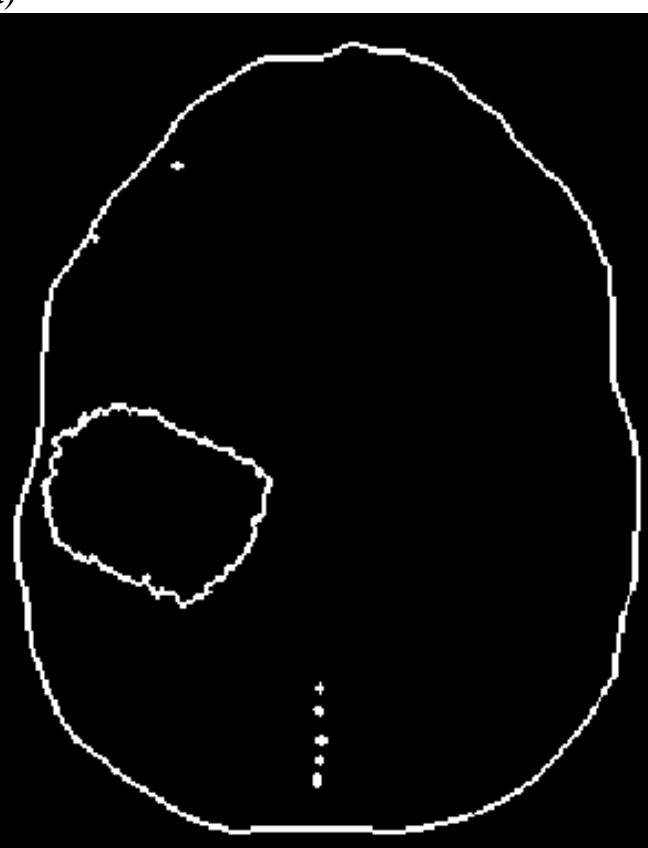

(c)

Figure 7. Mean Shift Gradient thresholding of a brain tumor image, without filtering step. 6(a) Histogram. 6(b) After thresholding. 6(c) Before thresholding

In Figure 8, the results related with groundtruth appear. Note that our algorithm achieved good results with no gaps. However, the obtained results by the Canny Edge Detector have 
International Journal of Soft Computing, Mathematics and Control (IJSCMC),Vol. 4, No. 2, May 2015

gaps. The range of threshold for the Canny Edge Detector was [0:0063; 0:0156], out of this interval the image becomes in oversegmentation or undersegmentation. The related results to real images are presented in Figure 9. Note the good obtained results, where so much edges as hemorrhage have been well delimited.

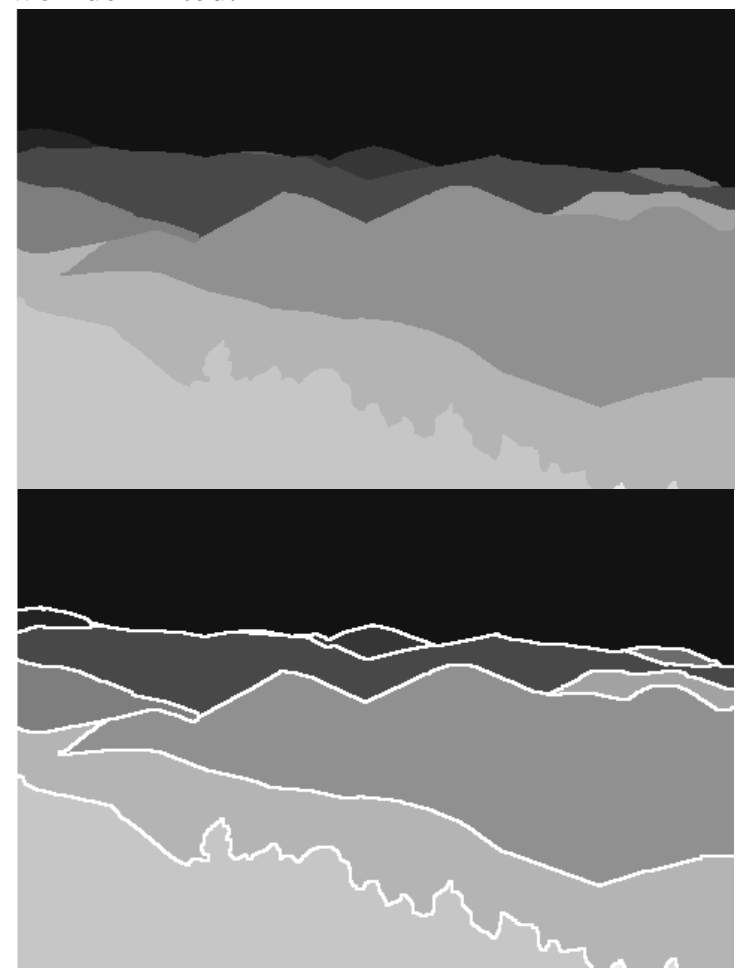

(a) Original image

(b) Edge superpose

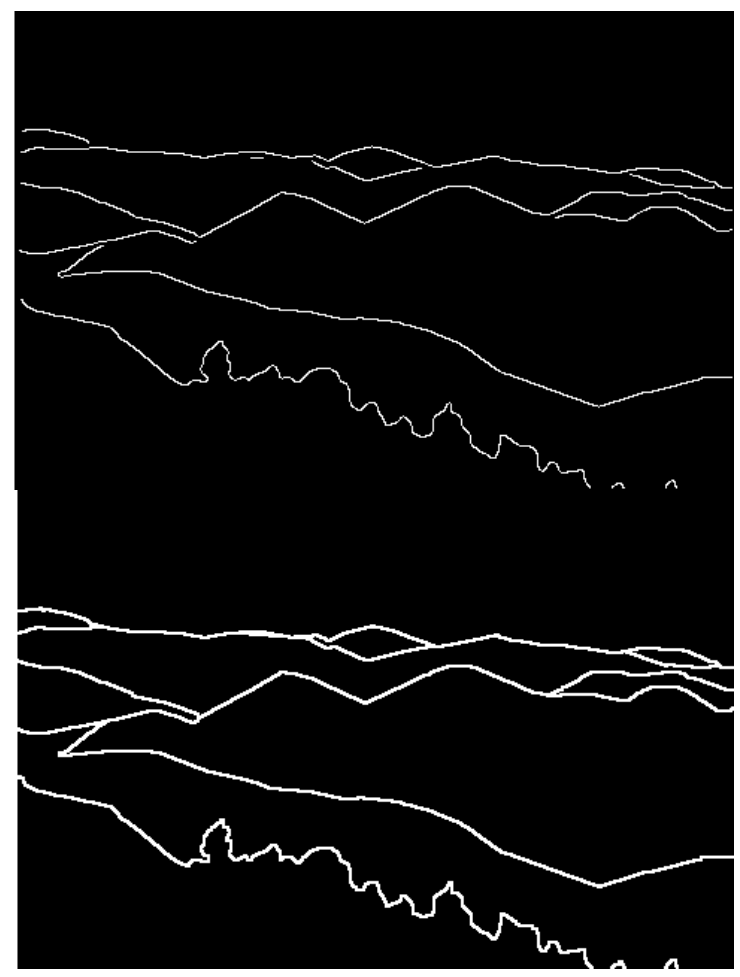


International Journal of Soft Computing, Mathematics and Control (IJSCMC),Vol. 4, No. 2, May 2015

(c) Canny

(d) MGRU

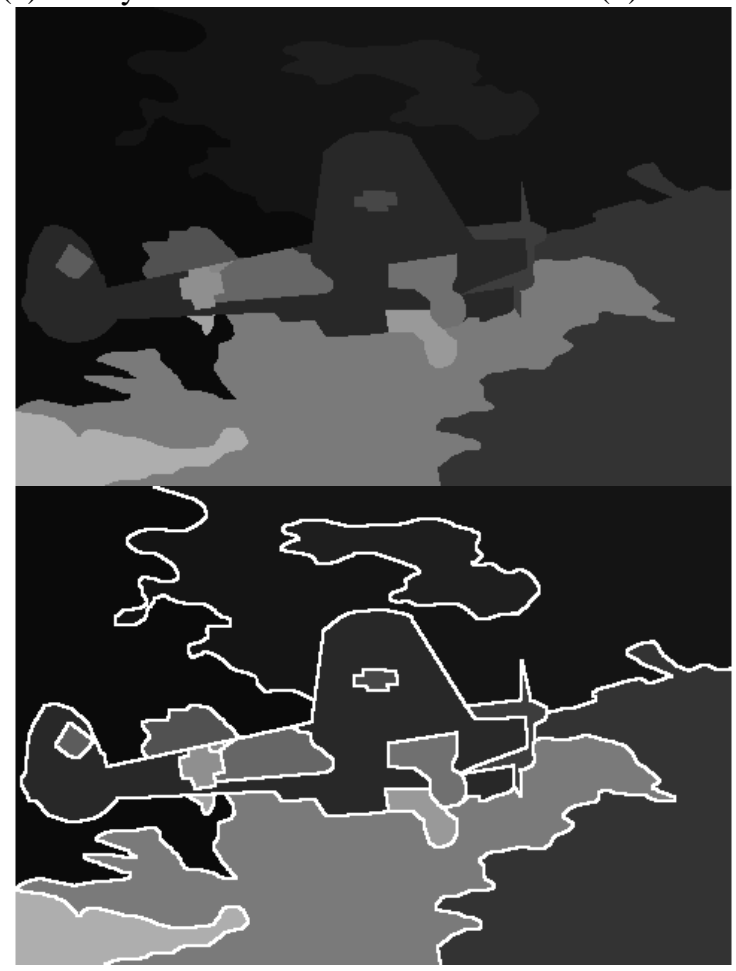

(e) Original image

(f) Edge superpose

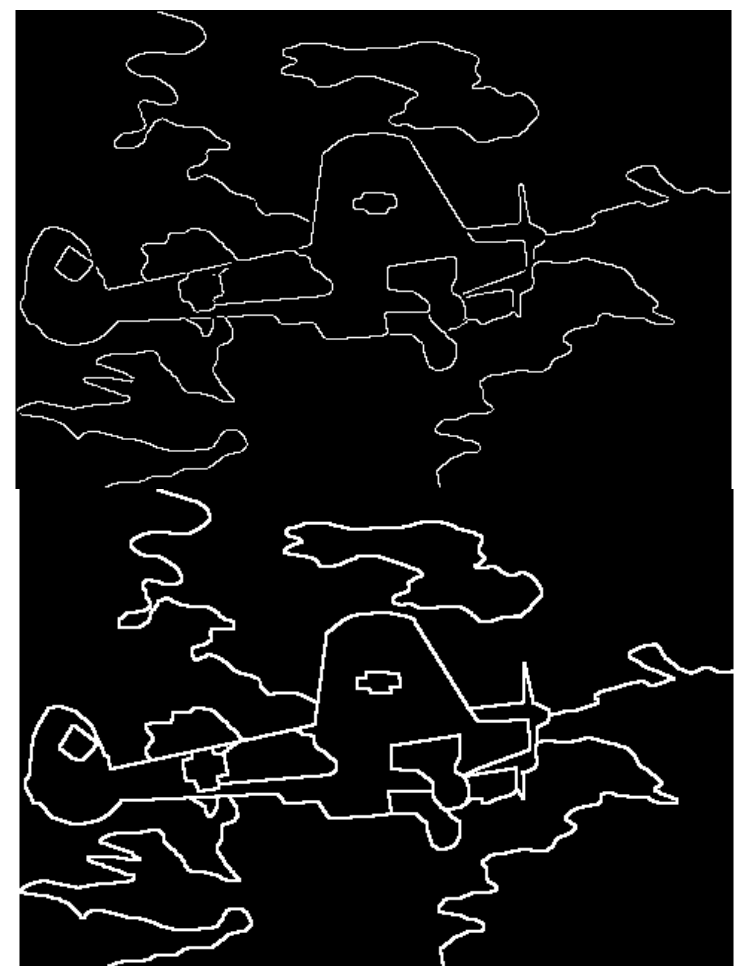

(g) Canny

(h) MGRU

Figure 8. Comparison with Canny Edge Detector. 
International Journal of Soft Computing, Mathematics and Control (IJSCMC),Vol. 4, No. 2, May 2015

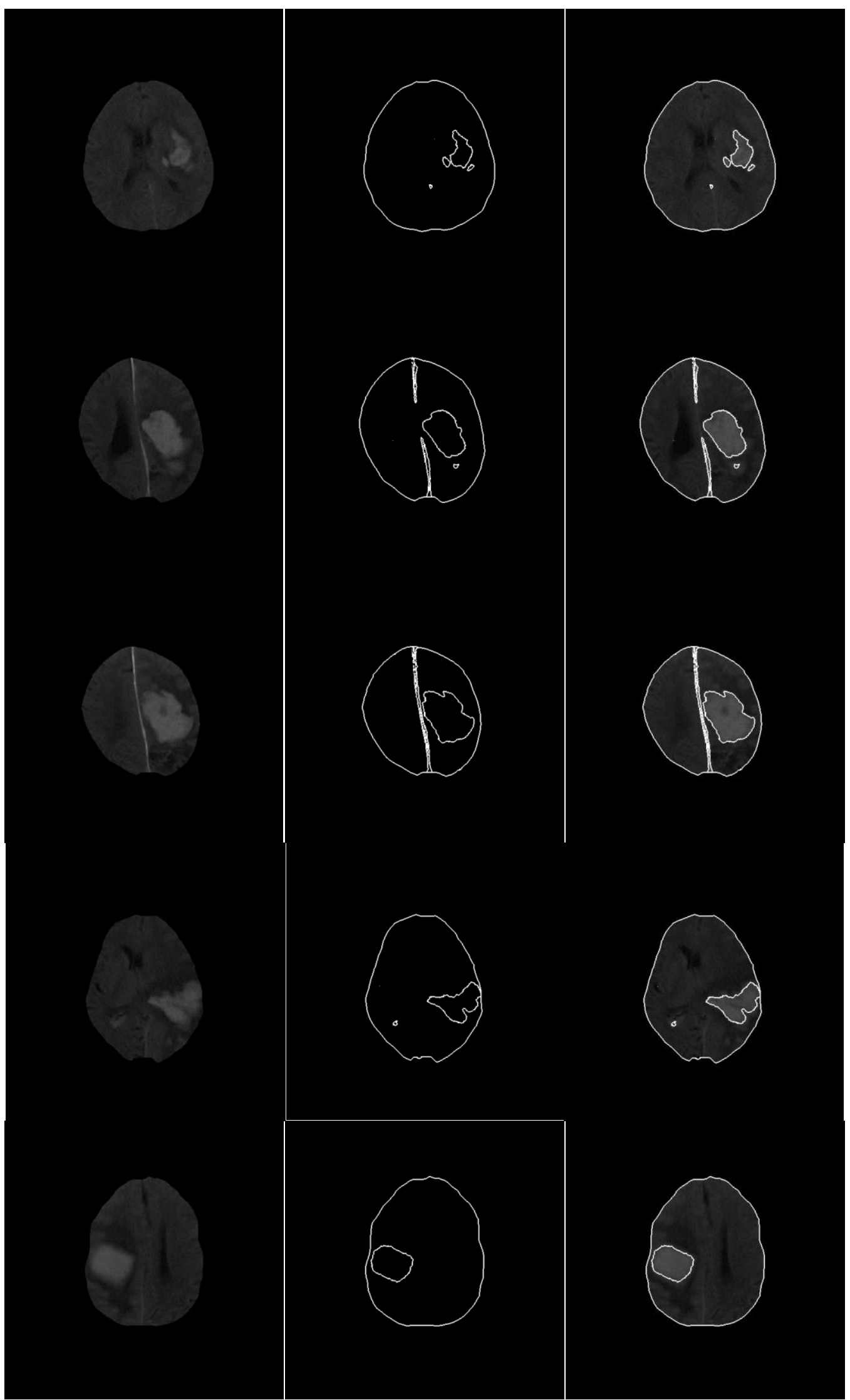


Figure 9. Result by applying our algorithm. 1st column: original image, 2nd column: edge image, 3th column: edge

\section{CONCLUSIONS} superpose.

In this paper, the theoretical aspects were exposed for the proposal of an edge detection algorithm. The theoretical aspects are related with the Ring Theory in images. This theory was applied to groundtruth and real images. Our method was compared with the method of the Canny edge detection for groundtruth images. Symmetrical ring units were utilized to highlight contour, and the Mean Shift gradient was used for the localization of edges. Several theorem concerning to the ring unit theory applied on images were exposed and proved. Through the obtained results was possible to see that, by using our method, continuous edges were obtained. In future works, the experimental results related to standard and real images will be shown. Also, we will study how to find automatically, the values of the threshold and the unit.

\section{REFERENCES}

[1] Chen, S. \& Wang, M., (2005) "Seeking multi-thresholds directly from support vectors for image segmentation", Neurocomputing, Vol. 67, No. 4, pp335-344.

[2] Cheng Y., (1995) "Mean Shift, Mode Seeking, and Clustering", IEEE Trans, Pattern Analysis and Machine Intelligence Neurocomputing, Vol. 17, No. 8, pp790-799..

[3] Comaniciu, D. I., (2000) "Nonparametric Robust Method for Computer Vision", Ph.D. Thesis, New Brunswick, Rutgers, The State University of New Jersey.

[4] Comaniciu, D. \& Meer, P., (2002) "Mean Shift: A Robust Approach toward Feature Space Analysis", IEEE Transaction on Pattern Analysis and Machine Intelligence, Vol. 24, No. 5.

[5] Domínguez, D. \& Rodríguez, R.: Use of the L (infinity) norm for image segmentation through Mean Shift filtering, International Journal of Imaging, Vol. 2, No. S09, pp81-93, 2009.

[6] Fukunaga, K. \& Hosteler, D. (1975) "The Estimation of the Gradient of a Density Function", IEEE Trans., Information Theory, No. 21, pp32-40.

[7] Garces, Y., Torres, E., Pereira, O., Perez, C. \& Rodriguez, R., (2013) "Stopping Criterion for the Mean Shift Iterative Algorithm", Progress in Pattern Recognition, Image Analysis, Computer Vision, and Applications, Springer, Lecture Notes in Computer Science, No. 8258, pp383-390.

[8] Garcés, Yasel; Torres, Esley; Pereira, Osvaldo \& Rodríguez, (2014) "Roberto Application of the Ring Theory in the Segmentation of Digital Images", International Journal of Soft Computing, Mathematics and Control, Vol. 3, No. 4.

[9] Guo, R. \& Pandit, S.M., (1998) Automatic threshold selection based on histogram modes and discriminant criterion, Machine Vision and Applications, No. 10, pp331-338.

[10] Grenier, T., Revol-Muller, C., Davignon, F., \& Gimenez, G., (2006) "Hybrid Approach for Multiparametric Mean Shift Filtering", Image Processing 2006, IEEE, International Conference, Atlanta, GA, Vol. 8, No. 11, pp1541-1544.

[11]J ohnson R.E, (1966) "University Algebra", Prentice-Hall, Engledwood Cliffs.

[12] Kuo H. W. and Sun Y.N., (2010) "Watershed segmentation with Automatic Altitude Selection and Region Merging Based on the Markov Random Field Model", International Journal of Pattern Recognition and Artificial Intelligence, Vol. 24, No. 1, pp153-171.

[13] Loncarics, S., (1998) "A survey of shape analysis techniques”, Pattern Recognition, Vol. 31, No. 8, pp983-1001, 1998

[14] Osuan-Encison, V., Cuevas, E. \& Sossa, H., (2013) "A comparison of nature inspired algorithms for multi-threshold image segmentation", Expert Systems with Applications, No. 40, pp12131219.

[15] Otsu, N., A threshold selection method from gray-level histograms, IEEE Transactions on Systems, Man and Cybernetics, Vol. 9, No. 1, pp62-66.

[16] Pal, N. R. \& Pal, S. K. (1993) A review on image segmentation techniques, Pattern Recognition, No. 26, pp. 1277-1294. 
[17] Reza, A.R. \& Brewer, V.E., (1989) “Edge Detection Using a Cyclic Ring of Integers Modulo 7”, IEEE, pp. 145-147.

[18] Rietman, E.A., Karp, R.L. \& Tuszynski J.A., (2011) "Review and application of group theory to molecular systems biology", Theoretical Biology and Medical Modelling, Vol. 8, No. 21.

[19] Rodriguez R. \& Suarez, A. G., (2006) “An Image Segmentation Algorithm Using Iteratively the Mean Shift", Image Analysis and Applications, Book Progress in Pattern Recognition, Book Series Lecture Notes in Computer Science Publisher Springer Berlin/Heidelberg, Vol. 4225/2006 pp326-335.

[20] Rodriguez R., (2008) "Binarization of medical images based on the recursive application of mean shift filtering: Another algorithm", Journal of Advanced and Applications in Bioinformatics and Chemistry, , Dove Medical Press Ltd, Vol. I, No. 1:12.

[21] Rodriguez, R., Suarez A. G. \& Sossa J. H., (2011) “A Segmentation Algorithm based on an Iterative Computation of the Mean Shift Filtering", Journal Intelligent and Robotic System, Vol. 63, No.3-4, pp447-463.

[22] Rodriguez, R., Torres E. \& Sossa J. H., (2012) "Image Segmentation via an Iterative Algorithm of the Mean Shift Filtering for Different Values of the Stopping Threshold", International Journal of Imaging and Robotics, Vol. 7, No. 6, pp1-19.

[23] Rodriguez, R., Torres E. \& Sossa J. H., (2011) "Image Segmentation based on an Iterative Computation of the Mean Shift Filtering for different values of window sizes", International Journal of Imaging and Robotics, Vol. 6, No. A11, pp1-19.

[24] Shannon C., A, (1948) "Mathematical Theory of Communication", Bell System Technology Journal, No. 27, pp370-423.

[25] Shen, D., Horace, H.S., (1997) "A Hopfield neural for adaptive image segmentation: An actice surface paradigm”, Pattern Recognition Letters, No. 18, pp37-48.

[26] Shen, C.\& Brooks, M. J., (2007) "Fast Global Kernel Density Mode Seeking: Applications to Localization and Tracking", IEEE Transactions on Image Processing, Vol. 16, No.5, pp14571469.

[27] Suyash, P. A. \& Ross T.W., (2006) "Higher-Order Image Statistics for Unsupervised, InformationTheoretic, Adaptive, Image Filtering", IEEE Transactions on Pattern Analysis and Machine Intelligence, Vol. 28, No. 3, pp364-376.

[28] Venkat, T., Govardhan, A. \& Badashah S. J., (2011) "Statistical Analysis for Performance Evaluation of Image Segmentation Quality Using Edge Detection Algorithms", International Journal of Advanced Networking and Applications, No 03, pp1184-1193.

[29] Wei, C. \& Kangling, F., (2008) "Multilevel thresholding algorithm based on particle swarm optimization for image segmentation", 27th Chinese control conference, Vol.67, No. 4, pp348351.

[30] Zhang, D. \& Lu G., (2003) "Review of shape representation and description techniques", Pattern Recognition Society, Published by Elsevier, No. 37, pp1- 19.

[31] Zhiwei, Y., Zhengbing, H., Huamin,. \& Hongwei CH., (2011) "Automatic threshold selection based on artificial bee colony algorithm", 3rd International Workshop on Intelligent Systems and Applications,

pp1-4. 
International Journal of Soft Computing, Mathematics and Control (IJSCMC),Vol. 4, No. 2, May 2015

\section{APPENDIX: THEOREMS AND PROOFS}

Theorem 1: Generators of $\mathbb{Z}_{n}$

Let $n \in \mathbb{Z}_{+}, \alpha \in \mathbb{Z}_{n}$ be such as $(\alpha, n)=1$, then $\alpha$ is a generator of $\mathbb{Z}_{n}$.

$(\alpha, n)=1$ means that $\alpha$ and $n$ are relatively prime.

Proof:

Let $\alpha, n \in \mathbb{Z}_{n},(\alpha, n)=1$ be $\Leftrightarrow \exists u, v \in \mathbb{Z}_{n}: u \alpha+v n=1$, then we obtain that $x=1 \cdot x=(u \alpha+v n) \cdot x=(u x) \alpha+(n x) v=(u x) \alpha$.

Let $k=u x$ be, then $x=k \alpha$, and this means that $\alpha$ is a generator of $\mathbb{Z}_{n}$.

Theorem 2: Injectivity of generators of $\mathbb{Z}_{n}$

Let $\alpha, x, y \in \mathbb{Z}_{n}$ be $x \neq y,(\alpha, n)=1 \Rightarrow \alpha x \neq \alpha y$.

Proof: Proof by Absurd

Let $\alpha x=\alpha y$ be, then $\alpha(x-y)=0$. Therefore $(\alpha, n)=1$, then $\alpha$ is generator of $\mathbb{Z}_{n}$, so the order of $\alpha$ is $n$. It follow that $(x-y)=k n=0 \Rightarrow(x-y)=0 \Rightarrow x=y$, and this is a contradiction because by hypothesis $x \neq y$.

Theorem 3: Equivalence relation of proportional images

Let the symbol $\sim$ represent the relation defined on a set of comparable images such that $G_{1} \sim G_{2}$ are proportional. Then, the relation between $G_{1}$ and $G_{2}$ is an equivalence relation.

Proof:

Let $G_{1}, G_{2}$ and $G_{3}$ be three images defined in a set of comparable images, then:

1) Symmetry

$G_{1}=1 * G_{1}$ therefore $G_{1}$ is related to itself, because $(1, \alpha)=1 \forall \alpha \in Z$.

2) Reflexivity

If $G_{1} \sim G_{2}$ then $\exists \alpha \in \mathbb{Z}_{n},(n, \alpha)=1: G_{1}=\alpha * G_{2}$, therefore

$G_{2}=1 * G_{2}=(n u+\alpha v) * G_{2}=u *\left(n * G_{2}\right)+v *\left(\alpha * G_{2}\right)=u * O+v * G_{1}=v * G_{1}$.

3) Transitivity

If $G_{1} \sim G_{2}, G_{2} \sim G_{3} \Rightarrow \exists \alpha_{1}, \alpha_{2} \in \mathbb{Z}_{n}:\left(\alpha_{1}, n\right)=1,\left(\alpha_{2}, n\right)=1$, so $G_{1}=\alpha_{1} * G_{2}, G_{2}=\alpha_{2} * G_{3}$ therefore

$G_{1}=\alpha_{1} * G_{2}=\alpha_{1} *\left(\alpha_{2} * G_{3}\right)=\left(\alpha_{1} \cdot \alpha_{2}\right) * G_{3}=\alpha * G_{3}$, where $\alpha=\alpha_{1} \cdot \alpha_{2}$.

But, it is necessary to prove that $\alpha=\alpha_{1} \cdot \alpha_{2}$ and $n$ are relatively prime integers, i.e., $\exists u, v \in \mathbb{Z}: 1=n u+\alpha v$.

If $\left(\alpha_{1}, n\right)=1,\left(\alpha_{2}, n\right)=1 \Rightarrow \exists u_{1}, u_{2} v, v_{2} \neq 0$ such as

$1=n u_{1}+\alpha_{1} v_{1}, 1=n u_{2}+\alpha_{2} v_{2}$, then

$\left(1-n u_{1}\right) / v_{1}=\alpha_{1}$ and $\left(1-n u_{2}\right) / v_{2}=\alpha_{2}$, so

$\alpha_{1} \cdot \alpha_{2}=\left(1-n u_{1}\right) / v_{1} \cdot\left(1-n u_{2}\right) / v_{2}=\left(1-n u_{1}\right) \cdot\left(1-n u_{2}\right) /\left(v_{1} \cdot v_{2}\right)$, 
International Journal of Soft Computing, Mathematics and Control (IJSCMC),Vol. 4, No. 2, May 2015

$\left(\alpha_{1} \cdot \alpha_{2}\right)\left(v_{1} \cdot v_{2}\right)=\left(1-n u_{1}\right) \cdot\left(1-n u_{2}\right)=1-n\left(u_{2}-u_{1}+n\left(u_{1} u_{2}\right)\right), \quad$ therefore $1=\left(\alpha_{1} \cdot \alpha_{2}\right)\left(v_{1} \cdot v_{2}\right)+n\left(u_{2}-u_{1}+n\left(u_{1} u_{2}\right)\right)$.

Consequently, if $u=n\left(u_{2}-u_{1}+n\left(u_{1} u_{2}\right)\right)$ and $v=\left(v_{1} \cdot v_{2}\right)$, therefore $\alpha=\alpha_{1} \cdot \alpha_{2}$ and $n$ are relatively prime integers.

Theorem 4: Bijective correspondence of proportional images

If two images are proportional, then their respective sets of gray levels are on bijective correspondence.

Proof:

Let $G_{1}, G_{2}$ be two proportional images defined in a set of comparable images, then $\exists \alpha \in \mathbb{Z}_{n}$ : $(\alpha, n)=1, G_{2}=\alpha * G_{1}$.

Let $I_{1}=\left\{i_{1} \in\left[0,2^{B}-1\right]: p_{i}^{(1)} \neq 0\right\}$ be the set of gray levels present in the image $G_{1}$, i.e., $I_{1}=i_{1}^{1}, \cdots, i_{1}^{m_{1}}$, where $m_{1}$ is the number of gray levels on $G_{1}$.

Let $I_{2}=\left\{i_{2} \in\left[0,2^{B}-1\right]: p_{i}^{(2)} \neq 0\right\}$ be the set of gray levels present in the image $G_{2}$, i.e., $I_{2}=i_{2}^{1}, \cdots, i_{2}^{m_{2}}$, where $m_{2}$ is the number of gray levels on $G_{2}$.

On the other hand, $I_{2}=\alpha * I_{1}=\left\{\alpha \cdot i_{1}^{1}, \cdots, \alpha \cdot i_{1}^{m_{1}}\right\} 1 \mathrm{~g}$, so it satisfies that $i_{2}^{r}=\alpha \cdot i_{1}^{r} \forall r \in I_{1}$.

Thefore, by theorems 1 and 2, the sets $I_{1}$ and $I_{2}$ are on bijective correspondence.

Theorem 5: Entropy of proportional images

If two images are proportional then have the same entropy.

Proof:

Let $G_{1}$ and $G_{2}$ be two proportional images defined in a set of comparable images then, $\exists \alpha \in \mathbb{Z}_{n},(\alpha, n)=1: G_{2}=\alpha * G_{1}$.

Let $E_{1}, E_{2}$ be their respective entropies of $G_{1}, G_{2}$, then: $E_{1}=-\sum_{i \in I_{1}} p_{i}^{1} \log _{2} p_{i}^{1}$ and $E_{1}=$ $-\sum_{i \in I_{1}} p_{i}^{2} \log _{2} p_{i}^{2}$.

Let $I_{1}=\left\{i_{1} \in\left[0,2^{B}-1\right]: p_{i}^{1} \neq 0\right\}$ be the set of gray levels present in the image $G_{1}$. Let $I_{2}=$ $\left\{i_{2} \in\left[0,2^{B}-1\right]: p_{i}^{(2)} \neq 0\right\}$ be the set of gray levels present in the image $G_{2}$.

$I_{2}=\alpha * I_{1} \Rightarrow i_{2}^{r}=\alpha \cdot i_{1}^{r} \forall r \in I_{1}$.

Equally, it there is a bijective correspondence between the images, and in addition, it there is a one-one correspondence among their sets of gray levels, then the values of the probabilities of occurrence of gray levels do not change. Only change the gray level of these pixels, so: $p_{i_{2}^{r}}^{2}=$ $p_{\alpha * i_{1}^{r}}^{1}=p_{i_{1}^{r}}^{1}$, therefore

$E_{2}=-\sum_{i \in I_{1}} p_{i_{2}^{r}}^{2} \log _{2} p_{i_{2}^{r}}^{2}=-\sum_{i \in I_{1}} p_{\alpha * i_{1}^{r}}^{1} \log _{2} p_{\alpha * i_{1}^{r}}^{1}=-\sum_{i \in I_{1}} p_{i_{1}^{r}}^{1} \log _{2} p_{i_{1}^{r}}^{1}=E_{1}$ 